\title{
No Change in Determining Crohn's Disease Recurrence or Need for Endoscopic or Surgical Intervention With Modification of the Rutgeerts Scoring System
}

\author{
Pauline Rivière, ${ }^{*, \neq}$ Séverine Vermeire, ${ }^{\ddagger}$ Marie Irles-Depe, ${ }^{*}$ Gert van Assche, ${ }^{\ddagger}$ Paul Rutgeerts, ${ }^{\ddagger}$ \\ Anthony de Buck van Overstraeten, ${ }^{\S}$ Quentin Denost, " Albert Wolthuis, ${ }^{\S}$ Andre D’Hoore, ${ }^{\S}$ \\ David Laharie, ${ }^{*}$ and Marc Ferrante ${ }^{\ddagger}$
} Hepatogastroenterology and Digestive Oncology, Haut-Lévêque Hospital, CHU de Bordeaux, Bordeaux, France; ' Department Haut-Lévêque Hospital, CHU de Bordeaux, Bordeaux, France

The postoperative endoscopic recurrence score, commonly referred to as the Rutgeerts score, was designed to predict clinical recurrence risk in Crohn's disease (CD) patients undergoing ileocolonic resection based on early endoscopic findings at the anastomosis and in the neoterminal ileum. ${ }^{1}$ In the pivotal publication, the i2 category, including aphthous lesions in the terminal ileum as well as ileocolonic anastomosis lesions, had a heterogeneous recurrence risk. Because anastomotic ulcers were suspected to be postsurgical ischemic lesions and less predictive of progressive disease, ${ }^{2}$ a modified Rutgeerts score (mRS) was proposed: i2a, lesions confined to the anastomosis $\pm<5$ isolated aphthous ulcers in the ileum; i2b, more than 5 aphthous ulcers in the ileum with normal mucosa in between, \pm anastomotic lesions. ${ }^{3,4}$

This study investigated the difference in clinical postoperative recurrence and need for endoscopic dilatation or new surgical intervention between $\mathrm{i} 2 \mathrm{a}$ and $\mathrm{i} 2 \mathrm{~b}$ endoscopic recurrence.

\section{Methods}

This retrospective study was conducted in the University Hospitals of Leuven (Belgium) and Bordeaux University Hospitals (France). Consecutive adult CD patients undergoing an ileocolonic resection with ileocolonic anastomosis between 2000 and 2013 evaluated by ileocolonoscopy within 1 year after the surgery and at least 3 years of follow-up after endoscopy were included.

One trained investigator (P.R.) revised endoscopic reports to attribute the mRS based on description of the lesions and images when available.

Clinical postoperative recurrence (CPOR) was defined as the occurrence of $\mathrm{CD}$ related symptoms associated to C-reactive protein $>5 \mathrm{mg} / \mathrm{L}$ and/or endoscopic recurrence $\geq \mathrm{i} 2 \mathrm{a}$ or radiologic evidence of neoterminal ileitis. When the recurrence was not
${ }^{*}$ Department of Gastroenterology and Hepatology, University Hospitals Leuven, KU Leuven, Leuven, Belgium; ${ }^{\ddagger}$ Department of of Abdominal Surgery, University Hospitals Leuven, KU Leuven, Leuven, Belgium; and "Department of Abdominal Surgery,

localized on the surgery site (eg, perianal disease, colitis, jejunitis), patients were considered as lost to follow-up at date of recurrence. New surgery included resection or stricturoplasty of the ileocolonic anastomosis. Surgical POR (sPOR) was the combination of endoscopic dilatation and new surgery.

Patients already experiencing cPOR at time of endoscopy were excluded for cPOR analyses. Statistical analyses were performed using R software version $\bullet \bullet \bullet Q 4$ (Survival package; R Foundation for Statistical Computing, Vienna, Austria).

\section{Results}

A total of 365 patients were included: $49 \%(n=180)$, median age 36 (interquartile range, 27-48) years, median follow-up 79 (interquartile range, 50-87) months, had an i2 Rutgeerts score, of whom $51 \%(n=91$ of 180$)$ had an i2a mRS and $49 \%(n=89$ of 180$)$ had an i2b mRS. No difference was observed between $i 2 a$ and $i 2 b$ patients regarding history of previous ileocolonic resection $(21 \%$ vs $32 \% ; P=.15)$, penetrating phenotype (36\% vs $33 \% ; P=.97$ ), prophylactic therapy after surgery $(20 \%$ vs $18 \% ; P=.91)$, or active smoking at endoscopy (30\% vs $23 \% ; P=.42$ ). Patients with mRS i2b received significantly more treatment initiation than did i2a patients after endoscopy (odds ratio, 3.3; 95\% confidence interval, 1.8-6.3; $P<.001$ ).

Comparing i2a and i2b patients, cPOR-free survival rates were not significantly different (log-rank $P=.70)$ (Figure $1 A$ ). In detail, similar cPOR-free survival rates were observed 1 year $(91.1 \%$ vs $90.7 \% ; P=.09)$ and 3 years $(76.5 \%$ vs $69.4 \% ; P=.31)$ after postoperative 

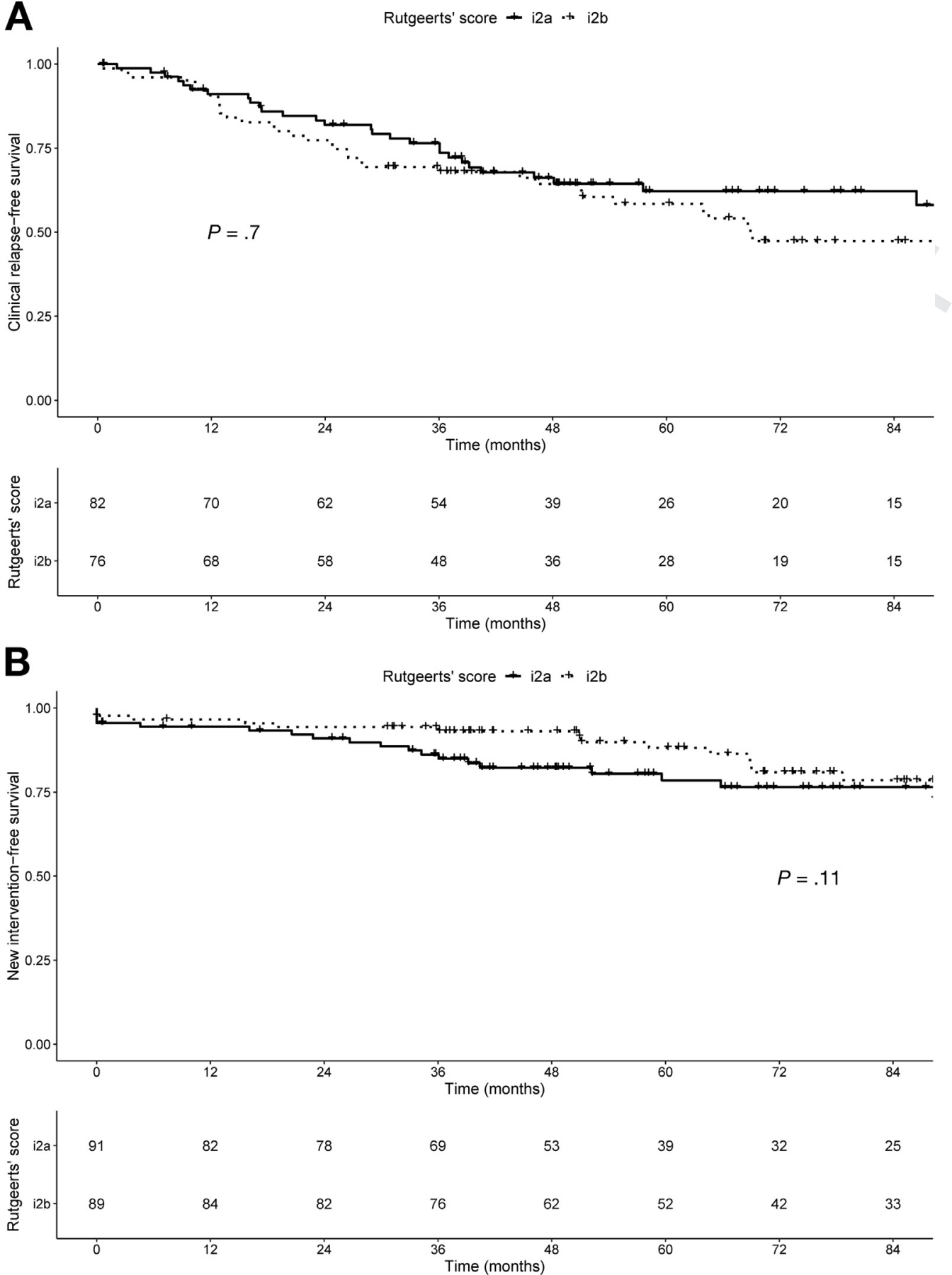

Figure 1. Outcomes after ileocolonic resection stratified by the modified Rutgeerts score at postoperative endoscopy. (A) Kaplan-Meier curves plotted from endoscopy to clinical postoperative recurrence. Clinical postoperative recurrence is defined as symptoms suggestive of Crohn's disease recurrence associated to objective criteria of disease activity (C-reactive protein $>5 \mathrm{mg} / \mathrm{L}$ or Rutgeerts score $>\mathrm{i} 2$ or radiologic terminal ileitis). Analysis restricted to patients not experiencing already clinical recurrence before postoperative endoscopy. (B) KaplanMeier curves plotted from endoscopy to surgical postoperative recurrence defined as endoscopic dilatation or refection of the ileocolonic anastomosis (all patients included).

endoscopy. Similarly, sPOR-free survival rates were not significantly different between $\mathrm{i} 2 \mathrm{a}$ and $\mathrm{i} 2 \mathrm{~b}$ patients (logrank $P=.11$ ) (Figure $1 B$ ): $94.5 \%$ and $96.6 \%(P=.45)$ at 1 year and $86.2 \%$ and $94.3 \%(P=.07)$ at 3 years, respectively. Of note, these findings were not modified by exclusion of patients receiving immediate change of therapy after endoscopy (log-rank $P=.17$ for cPOR and $P=.30$ for $\mathrm{SPOR}$ ).

In multivariate analysis, cPOR was independently associated with smoking at endoscopy (hazard ratio, 1.7; 95\% confidence interval, 1.0-2.8; $P=.05$ ) and the absence of a laterolateral anastomosis (hazard ratio, 1.8; 95\% confidence interval, $1.0-3.1 ; P=.05$ ).

\section{Discussion}

In this retrospective study, no difference was observed in terms of clinical postoperative recurrence and need for endoscopic or surgical intervention between patients with i2a or i2b endoscopic recurrence. Our data confirm on a larger cohort findings from a previously published retrospective study. ${ }^{5}$

The major limitation of our study is its retrospective setting. Ileocolonoscopy was not performed routinely in operated patients in the early years of the cohort. Some patients were excluded because of shorter follow-up duration. These 2 factors could have led to a selection 
bias toward more severe patients. Two centers were involved in this work; however, no difference was observed when comparing patients' profiles, prophylactic therapy, or treatment modifications (data not shown). Interobserver reproducibility of the RS has been shown to be suboptimal, especially for the i2 subscore. ${ }^{6}$ To limit this variability, each endoscopy was revised to reassess the mRS by 1 unique investigator. Objective outcomes defining CPOR were retained and only patients having a long-term follow-up have been included to restrict the observational bias.

Endoscopic recurrence RS i2a and i2b do not differ in terms of clinical recurrence-free survival and need for a new intervention.

\section{References}

1. Rutgeerts P, Geboes K, Vantrappen G, Beyls J, Kerremans R, Hiele M. Predictability of the postoperative course of Crohn's disease. Gastroenterology 1990;99:956-963.

2. Vuitton L, Marteau P, Sandborn WJ, et al. IOIBD technical review on endoscopic indices for Crohn's disease clinical trials. Gut 2016;65:1447-1455.

3. Domènech $\mathrm{E}$, Mañosa $\mathrm{M}$, Bernal I, et al. Impact of azathioprine on the prevention of postoperative Crohn's disease recurrence: results of a prospective, observational, long-term follow-up study. Inflamm Bowel Dis 2008;14:508-513.

4. Gecse K, Lowenberg M, Bossuyt $P$, et al. Sa1198 agreement among experts in the endoscopic evaluation of postoperative recurrence in Crohn's disease using the Rutgeerts score. Gastroenterology 2014;146:S227.
5. Bayart P, Duveau N, Nachury M, et al. lleal or anastomotic location of lesions does not impact rate of postoperative recurrence in Crohn's disease patients classified i2 on the Rutgeerts score. Dig Dis Sci 2016;61:2986-2992.

6. Marteau $\mathrm{P}$, Laharie $\mathrm{D}$, Colombel J-F, et al. Interobserver variation study of the rutgeerts score to assess endoscopic recurrence after surgery for Crohn's disease. J Crohns Colitis 2016; 10:1001-1005.

\section{Reprint requests}

Address requests for reprints to: Marc Ferrante, Department of Gastroenterology and Hepatology, University Hospitals Leuven / KU Leuven, Herestraat 49, B3000 Leuven, Belgium. e-mail: marc.ferrante@uzleuven.be; fax: (32) 1634 4419.

\section{Acknowledgments}

Séverine Vermeire, Gert Van Assche, and Marc Ferrante are senior clinical in- Q6 vestigators of the Research Foundation - Flanders (FWO).

Conflicts of interest

These authors disclose the following: Séverine Vermeire has received grant Q3 support from AbbVie, MSD, Pfizer and Takeda; received speaker fees from AbbVie, MSD, Takeda, Ferring, Dr. Falk Pharma, Hospira, Pfizer Inc and Tillots; and served as a consultant for AbbVie, MSD, Takeda, Ferring, Genentech/Roche, Shire, Pfizer Inc, Galapagos, Mundipharma, Hospira, Celgene, Second Genome, and Janssen. Gert Van Assche received financial support for research from Abbvie, MSD; lecture fees from Janssen, Takeda, Ferring, MSD, Abbvie; and consultancy fees from Abbvie, MSD, Takeda

Paul Rutgeerts received consultant or advisory boards or lectures fees from Johnson \& Johnson; MSD; UCB; AbbVie; Takeda; Genentech; Bristol-Myers Squibb; Tillotts Pharma; Parexel; Quintiles; Robarts; Amgen/Medimmune/ AstraZeneca. David Laharie has received board or lectures fees from Abbvie, Celgene, Ferring, Janssen, MSD, Novartis, Pfizer, Roche and Takeda. Marc Ferrante has received speaker's fee from Abbvie, BoehringerIngelheim, Chiesi, Ferring, Janssen, MSD, Pfizer, Takeda, Tillotts, Zeria consultancy fees from Abbvie, Boehringer-Ingelheim, Ferring, Janssen, MSD, Pfizer, and Takeda, and a research grant from Janssen and Takeda. The remaining authors disclose no conflicts. 